OPEN ACCESS

Edited by:

Nadia Mores,

Catholic University of the Sacred

Heart, Italy

Reviewed by:

Francesco Varone,

Catholic University of the Sacred

Heart, Italy

Antonio Molino

University of Naples Federico II, Italy

*Correspondence: Hiroaki lkesue ikesue@kcho.jp

Specialty section:

This article was submitted to

Respiratory Pharmacology,

a section of the journal

Frontiers in Pharmacology

Received: 25 January 2020

Accepted: 16 October 2020

Published: 26 November 2020

Citation:

Satsuma $Y$, Ikesue $H$, Kusuda K, Maeda M, Muroi N, Mori R, Kogo M, Hirabayashi R, Nagata K, Nakagawa A,

Tachikawa R, Tomii $K$ and Hashida $T$ (2020) Effectiveness of

Pharmacist-Physician Collaborative

Management for Patients With

Idiopathic Pulmonary Fibrosis

Receiving Pirfenidone.

Front. Pharmacol. 11:529654.

doi: 10.3389/fphar.2020.529654

\section{Effectiveness of Pharmacist-} Physician Collaborative Management for Patients With Idiopathic Pulmonary Fibrosis Receiving Pirfenidone

\author{
Yukari Satsuma ${ }^{1}$, Hiroaki lkesue ${ }^{1 *}$, Kaori Kusuda ${ }^{1}$, Mami Maeda $^{1}$, Nobuyuki Muroi ${ }^{1}$, \\ Ryobu Mori ${ }^{2}$, Mariko Kogo ${ }^{2}$, Ryosuke Hirabayashi ${ }^{2}$, Kazuma Nagata ${ }^{2}$, Atsushi Nakagawa ${ }^{2}$, \\ Ryo Tachikawa ${ }^{2}$, Keisuke Tomii ${ }^{2}$ and Tohru Hashida ${ }^{1}$ \\ ${ }^{1}$ Department of Pharmacy, Kobe City Medical Center General Hospital, Kobe, Japan, ${ }^{2}$ Department of Respiratory Medicine, \\ Kobe City Medical Center General Hospital, Kobe, Japan
}

Background: Pirfenidone is an anti-fibrotic agent used to treat patients with idiopathic pulmonary fibrosis (IPF). Managing adverse drug events and ensuring compliance with pirfenidone treatment for a prolonged period are important to reduce the rate of disease progression. To maximize the benefits of pirfenidone treatment, we established and evaluated an ambulatory care pharmacy practice, a model of pharmacist-physician collaborative management, for patients receiving pirfenidone.

Methods: We conducted a retrospective chart review of 76 consecutive patients treated with pirfenidone in the Kobe City Medical Center General Hospital, Japan, between January 2012 and January 2019. The first group (61 patients) received pirfenidone treatment as conventional management, whereas the second group (15 patients) started pirfenidone based on collaborative pharmacist-physician management. The drug discontinuation rate and time to drug discontinuation were compared between the groups. To analyze factors associated with pirfenidone discontinuation, we used a multivariate Cox regression analysis to evaluate the baseline characteristics of patients, including those receiving the collaborative management. Clinical outcomes were compared using a propensity score matched analysis.

Results: In the collaborative management group, pharmacists made 56 suggestions, including suggestions for supportive care (51 suggestions), to the physicians. Among these suggestions, 52 were accepted by the physicians. The discontinuation rates at 3 [6.7\% (1/15) vs. 26.2\% (16/61)] and 6 [9.1\% (1/11) vs. 36.1\% (22/61)] months were lower in the collaborative management group than in the conventional management group. Multivariate analysis revealed that collaborative management [hazard ratio $(\mathrm{HR})$ $0.34,95 \% \mathrm{Cl} 0.08-0.96, p=0.041$ ] and predicted baseline forced vital capacity $<60 \%$ ( $\mathrm{HR} 2.13,95 \% \mathrm{Cl} 1.17-3.85, p=0.015)$ were significantly associated with pirfenidone discontinuation. The time to drug discontinuation was also significantly longer in the collaborative management group than in the conventional management group ( $p=$ 0.034, log-rank test). Propensity score matched analysis confirmed a significant 
correlation between collaborative management and drug discontinuation time (HR $0.20,95 \% \mathrm{Cl} 0.03-0.84, p=0.027)$.

Conclusions: We established an ambulatory care pharmacy practice for out-patients with
IPF receiving pirfenidone. The results suggest that collaborative management may help
prevent pirfenidone discontinuation compared with conventional management.

Keywords: idiopathic pulmonary fibrosis, pirfenidone, pharmacist, physician, collaborative management, ambulatory care pharmacy practice

\section{INTRODUCTION}

Idiopathic pulmonary fibrosis (IPF), the most common form of idiopathic interstitial pneumonia (IIP), is a progressive lung disease characterized by chronic refractory cough, shortness of breath, and exercise limitation (Ley et al., 2011; Lederer and Martinez, 2018). The prognosis of patients with IPF is poor, with a median survival of $2-5$ years after diagnosis (Ley et al., 2011; Raghu et al., 2015; Homma et al., 2018; Lederer and Martinez, 2018). Two anti-fibrotic agents, pirfenidone and nintedanib, are currently approved for the treatment of IPF in several countries (Raghu et al., 2015; Homma et al., 2018). The clinical efficacy of pirfenidone in patients with IPF has been confirmed in clinical trials in Japan (Azuma et al., 2005; Taniguchi et al., 2010) and in multinational randomized clinical trials (Noble et al., 2011; King et al., 2014). Continuing pirfenidone for a prolonged period is important to reduce the rate of disease progression. The most common adverse drug events (ADEs) associated with pirfenidone are gastrointestinal tract- and skin-related events (Lancaster et al., 2016; Noble et al., 2016). The management of ADEs is essential because these symptoms can lead to discontinuation of pirfenidone treatment by patients (Ogura et al., 2015; Hughes et al., 2016; Lancaster et al., 2017; Cottin et al., 2018).

Studies have reported the usefulness of patient-centered, multidisciplinary team care, which provides support, education, and empowerment to patients with IPF receiving pirfenidone (Duck et al., 2015; Ferrara et al., 2018). We assembled a multidisciplinary IIP support team comprising healthcare professionals, including physicians, pharmacists, nurses, dietitians, physical therapists, and social workers, to support patients with IIP. To support the functions of this team, we established an ambulatory care pharmacy practice to provide pharmacist-physician collaborative management of out-patients with IPF receiving anti-fibrotic therapy in September 2017. The main purpose of the collaborative management was to prevent early discontinuation of anti-fibrotic agents. Therefore, pharmacists provided information to physicians regarding appropriate prescriptions and education to patients to minimize ADEs and enhance adherence to anti-fibrotic agents. In the present study, we evaluated the usefulness of this management system for patients receiving pirfenidone.

\section{MATERIALS AND METHODS Establishing Pharmacist-Physician Collaborative Management of Pirfenidone Treatment for Out-Patients With Idiopathic Pulmonary Fibrosis}

We constructed a multidisciplinary IIP support team composed of healthcare professionals, including physicians, pharmacists, nurses, dietitians, physical therapists, and social workers, in the Kobe City Medical Center General Hospital, Japan. Initially, we prepared a patient education book, which helped to develop consensus among the healthcare professionals regarding the treatment and care of patients with IPF and to provide education to patients. We then established an ambulatory care pharmacy practice, comprising the pharmacist-physician collaborative management of out-patients with IPF receiving antithrombotic therapy, in September 2017. The objectives were to provide information to physicians for appropriate prescriptions and to patients to minimize ADEs and drug-drug interactions, to enhance the adherence of patients to treatment and maximize the effectiveness of anti-fibrotic therapies.

A patient flow diagram, including the ambulatory care pharmacy practice, is shown in Figure 1. When pirfenidone was initially prescribed, the physician advised the patient to visit the ambulatory care pharmacy practice after their clinical examination. In the ambulatory drug consultation room, a pharmacist educated the patients using the patient education book as follows: 1) initial dosage and time of dose; 2) standard titration schedule of pirfenidone; 3) symptoms and management of pirfenidone-associated ADEs, such as gastrointestinal events and photosensitivity; and 4) drug-drug interactions. The pharmacist also queried the patient about concomitant medications and supplements to avoid any drug-drug interactions. At the second visit or later, the patient visited the pharmacy service before being examination by the physician. The pharmacist assessed: 1) any ADEs and their grade according to the National Cancer Institute Common Terminology Criteria for Adverse Events version $4.0 ; 2$ ) whether the patient was able to take the medication for ADEs appropriately; 3) changes in any concomitant medications or supplements; and 4) adherence to pirfenidone, which was determined by asking the patient how many of the prescribed tablets remained. The pharmacist repeatedly educated the patient about the management of ADEs. The patient was allowed to ask the pharmacist any 


\section{Conventional care}

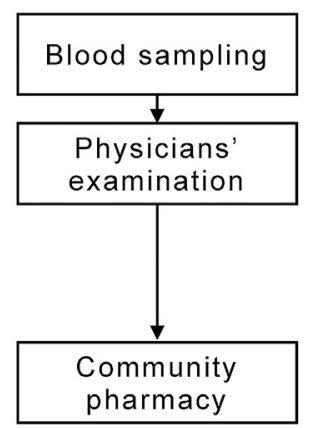

Pharmacist-physician collaborative management
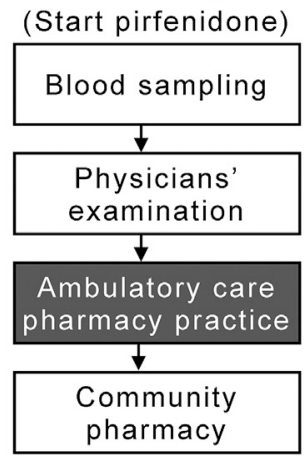

$\left(2^{\text {nd }}\right.$ visit or later)

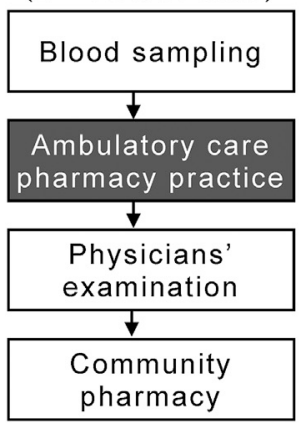

FIGURE 1 | Flow diagram of patients in the pharmacist-physician collaborative management and conventional groups. The collaborative management was initiated in September 2017; thereafter, 15 consecutive patients (the collaborative management group) were collaboratively managed by physicians and pharmacists. From January 2015 to August 2017, 61 patients received conventional care with pirfenidone (the conventional group).

questions directly in the ambulatory care pharmacy practice or by telephone. Per requirement, based on their assessment, the pharmacist suggested prescriptions to the physician. The patient then received the medication, including pirfenidone, from the community pharmacies.

The ambulatory care pharmacy practice comprised three clinical pharmacists who also provided clinical pharmacy service for in-patients in clinical wards. Our pharmacy practice was provided for all patients for the first 3 months after the start of pirfenidone therapy. If the patients wished to continue the service, they could visit the ambulatory care pharmacy practice any time before being examined by a physician. The ambulatory care pharmacy practice usually required $20 \mathrm{~min}$ per outpatient for a face-to-face consultation and an additional $5 \mathrm{~min}$ to enter the medical chart into an electronic medical record system to share the patients' information with the IPF support team.

\section{Patients}

We conducted a retrospective chart review of 76 consecutive Japanese patients with IPF prescribed pirfenidone between January 2015 and January 2019. All subjects received pulmonary care in the Kobe City Medical Center General Hospital, Japan. Pharmacist-physician collaborative management of patients receiving pirfenidone treatment was initiated in September 2017; thereafter, 15 consecutive patients (the collaborative management group) were managed collaboratively by physicians and pharmacists. From January 2015 to August 2017, 61 patients received conventional care with pirfenidone (the conventional group). Patients aged at least 20 years, diagnosed with pulmonary fibrosis by their primary pulmonologist during routine clinical care, and prescribed pirfenidone were included. One patient who received perioperative pirfenidone only (Iwata et al., 2016) was excluded. This study was conducted in accordance with the Declaration of Helsinki. The study protocol was approved by the Institutional Review Board of the Kobe City Medical Center General Hospital (No. h190401).
In Japan, the approved dosage of pirfenidone is $1,800 \mathrm{mg} /$ day (Azuma et al., 2005; Homma et al., 2018). In accordance with the package insert of pirfenidone in Japan, one $200 \mathrm{mg}$ pirfenidone tablet is administered orally three times a day (t.i.d.) after meals for the first 2 weeks $(600 \mathrm{mg} /$ day), two tablets t.i.d. for the next 2 weeks $(1,200 \mathrm{mg} /$ day $)$, and three tablets t.i.d. thereafter (1,800 mg/day). Physicians adjusted the dose-escalation schedule by observing each patient's condition. In the conventional care group, supportive care medicines, including domperidone, metoclopramide, and mosapride, were prescribed at the physicians' discretion. In contrast, all patients in the collaborative management group were prescribed domperidone $5 \mathrm{mg}$ t.i.d. orally at the onset of pirfenidone therapy (Costabel et al., 2014); the subsequent domperidone dose was prescribed at the physicians' discretion based on suggestions made by the pharmacists.

\section{Outcome Measures and Data Collection}

The primary objective of this study was to evaluate the effect of pharmacist-physician collaborative management on pirfenidone discontinuation in patients with IPF. The secondary objectives included the adherence rate and suggestions made to physicians by the pharmacists in the collaborative management group. To assess adherence to pirfenidone, the pharmacists queried patients about the remaining number of pirfenidone tablets at each visit for the first 3 months after the start of treatment. The adherence rate (\%) was calculated as follows (number of tablets taken)/ (number of tablets prescribed) $\times 100$. We also evaluated factors associated with pirfenidone discontinuation owing to ADEs in the conventional management group. All data were collected from the electronic medical records. The data cut-off date was January 31, 2019.

\section{Statistics}

Categorical data are displayed as the number of patients (n) and the respective relative frequencies. Values were compared using Fisher's exact test. For continuous data, median [interquartile range (IQR)] values are presented. Differences between groups 
were analyzed using Wilcoxon rank sum test. To analyze factors associated with pirfenidone discontinuation, a single predictor Cox regression analysis was performed using patients' age, sex, predicted baseline forced vital capacity (FVC), body mass index (BMI), smoking status, and pharmacist-physician collaboration management as independent variables. Significant factors in the univariate analysis were evaluated as potential covariates in the subsequent multivariable Cox regression analysis. The time to drug discontinuation was estimated using the Kaplan-Meier method and compared using the log-rank test. Patients who were still taking pirfenidone and were free from progression were censored at the last follow-up.

To adjust for the other baseline factors, a sensitivity analysis was conducted by propensity score matching. We estimated the propensity score by modeling the probability of being in the conventional management group vs. the collaborative management group. The following variables were included in the regression model: age, body weight, smoking status, concomitant use of prednisolone, FVC, and DLco. A 1:1 matching (without replacement) in the two treatment groups was achieved using the nearest neighbor method with a 0.25 -width caliper of SD of the logit of propensity scores to reduce bias by these potential confounding factors. The matched data were analyzed to confirm the robustness of the primary analysis results. Data were analyzed using JMP 13.2.1 (SAS Institute Inc., Cary NC, United States). The results with a $p$-value of $<0.05$ were considered statistically significant.

\section{RESULTS}

\section{Baseline Characteristics of Patients}

Between January 2015 and August 2017, 61 consecutive patients with IPF were prescribed pirfenidone treatment (the conventional group). After establishing the ambulatory care pharmacy practice, between September 2017 and January 2019, 15 consecutive patients with IPF were prescribed pirfenidone (the collaborative management group). The baseline characteristics of the 74 eligible patients are summarized in Table 1. There were no significant differences in the proportions of patients who were male ( $80.0 \mathrm{vs.} 54.2 \%, p=$ 0.138 ), ex-smokers (73.3 vs. $54.2 \%, p=0.245$ ), using home oxygen therapy ( 13.3 vs. $11.9 \%, p=1.000)$, or concomitantly receiving prednisolone ( 46.7 vs. $32.2 \%, p=0.367$ ) between the collaborative management and conventional groups. Similarly, baseline age, body weight, BMI, FVC, and 6-min walk distance were not significantly different between the groups. The median (IQR) predicted FVC (\%) in the collaborative management and conventional groups was $71.8(64.4-85.0)$ and 67.6 (53.8-85.0), respectively. The proportion of patients with a predicted FVC of $<60 \%$ was not different between the groups [20.0\% (3/15) vs. $37.3 \%(22 / 59), p=0.240]$. The baseline values of predicted diffusing capacity of carbon monoxide (DLco, \%) were not evaluated in 24 and three patients, in the conventional and the collaborative management group, respectively. The median (IQR) predicted DLco (\%) of the remaining patients was significantly lower in the collaborative management group than in the
TABLE 1 | Patient characteristics.

\begin{tabular}{|c|c|c|c|}
\hline & $\begin{array}{l}\text { Collaborative } \\
\text { management } \\
(n=15)\end{array}$ & $\begin{array}{l}\text { Conventional } \\
(n=61)\end{array}$ & $P$-value \\
\hline Age, years & $76(71-80)$ & $74(68-80)$ & 0.414 \\
\hline Male, n (\%) & $12(80.0)$ & $34(55.7)$ & 0.139 \\
\hline Body weight, kg & $58.5(51.5-65.0)$ & 57.6 (49.0-66.0) & 0.548 \\
\hline $\mathrm{BMl}, \mathrm{kg} / \mathrm{m}^{2}$ & $22.7(19.8-24.8)$ & $22.3(20.2-24.1)$ & 0.912 \\
\hline \multicolumn{4}{|l|}{ Smoker, n (\%) } \\
\hline Ex & $11(73.3)$ & $34(55.7)$ & 0.254 \\
\hline Never & $4(26.7)$ & 27 (44.3) & \\
\hline Home oxygen therapy, n (\%) & 2 (13.3) & $7(11.5)$ & 1.000 \\
\hline Prednisolone use, n (\%) & $7(46.7)$ & $20(32.8)$ & 0.372 \\
\hline \multicolumn{4}{|l|}{ Pulmonary function tests } \\
\hline FVC (L) & $2.3(1.6-2.7)$ & $1.9(1.5-2.5)$ & 0.464 \\
\hline FVC (\% pred) & $71.8(64.4-85.0)$ & $67.6(53.8-87.0)$ & 0.676 \\
\hline DLco (\% pred) & $51.8(42.5-62.2)^{\mathrm{a}}$ & $63.3(56.3-85.0)^{\mathrm{b}}$ & 0.031 \\
\hline 6-min walk distance, $\mathrm{m}$ & $413(256-485)^{\mathrm{C}}$ & $445(375-500)^{d}$ & 0.280 \\
\hline
\end{tabular}

$D L C O$, diffusing capacity of carbon monoxide; FVC, forced vital capacity. Continuous data are shown as the median (interquartile range). Fisher's exact test and Wilcoxon rank sum tests were used to compare categorical and continuous data, respectively. ${ }^{a}$ Missing data from 3 patients.

${ }^{b}$ Missing data from 24 patients.

${ }^{c}$ Missing data from 1 patient.

${ }^{d}$ Missing data from 14 patients.

conventional group $[51.8(42.5-62.2)$ vs. $63.3(56.3-85.0)$, $p=0.031]$

\section{Effect of Pharmacist-Physician Collaboration Management on Pirfenidone Discontinuation}

Pirfenidone discontinuation rates at 3, 6, and 12 months are shown in Supplementary Figure S1. In the collaborative management group, the observation period for four and six patients was less than 6 and 12 months, respectively. Three patients discontinued pirfenidone owing to ADEs (grade 2 photosensitivity at 2 months, grade 2 rash at 9 months, and grade 1 photosensitivity at 11 months) within the first 12 months. In contrast, among the 61 patients in the conventional group, 21 discontinued pirfenidone owing to ADEs (13 patients, nausea or anorexia; 3, malaise; 2, hepatotoxicity; and 3, other events) and 10 patients discontinued owing to an acute exacerbation of IPF or disease progression, within the first 12 months. One patient was transferred to another hospital after 11.3 months of treatment. The rates of discontinuation at $3[6.7(1 / 15)$ vs. $26.2 \%(16 / 61), p=0.167]$ and 6 [9.1 (1/11) vs. $36.1 \%(22 / 61)$, $p=0.093]$ months were lower in the collaborative management group than in the conventional group; however, the difference was not significant.

To evaluate the effect of pharmacist-physician collaborative management on the discontinuation of pirfenidone, we conducted univariate and multivariate analyses using a Cox proportional hazards model. The results revealed that collaborative management [hazard ratio (HR) $0.34,95 \% \mathrm{CI}$ $0.08-0.96, p=0.041$ ] and a predicted baseline FVC of $<60 \%$ (HR 2.13, 95\% CI 1.17-3.85, $p=0.015$ ) were significantly 
associated with pirfenidone discontinuation (Table 2). The Kaplan-Meier curve for the continuation of pirfenidone therapy is shown in Figure 2. The time to drug discontinuation was significantly longer in the collaborative management group than in the conventional group $(p=0.034$, log-rank test). In contrast, the prescription of antiemetics did not significantly decrease the rate of pirfenidone discontinuation owing to ADEs in the conventional group (Supplementary Table S1).

We also performed propensity score matching to balance patient characteristics between the collaborative management and conventional groups. There were no significant differences in the factors between the two groups (Table 3). In the propensity score-matched cohorts, the median time to drug discontinuation was significantly longer in the collaborative management group than in the conventional group [not reached (NR) (95\% CI $1.8-\mathrm{NR}$ ) vs. 2.9 months (95\% CI $0.1-\mathrm{NR}$ ), $p=0.028$, log-rank test], and there was a significant association between collaborative management and time to drug discontinuation (HR 0.20, 95\% CI 0.03-0.84, $p=0.027$ ).

\section{Activities of Pharmacists in the Ambulatory Care Pharmacy Practice}

In the collaborative management group, the pharmacists made a total of 56 suggestions to the physicians (Table 4). Among these suggestions, the required number of supportive care medicines that were previously prescribed (38 suggestions) was the most frequent. The suggestions also comprised additional supportive care (13 suggestions), including antiemetics or photosensitivity prevention, and others (5 suggestions). Among the 56 suggestions, 52 (92.9\%) were accepted by the physicians.

The pharmacists assessed patients' adherence to pirfenidone at each visit to the ambulatory care pharmacy practice. The results revealed that the median adherence of patients to pirfenidone for the first 3 months was $99.4 \%$ (98.4-100\%).

TABLE 2 | Univariate and multivariate Cox regression models for drug discontinuation.

\begin{tabular}{|c|c|c|c|c|}
\hline \multirow[t]{2}{*}{ Factor } & \multicolumn{2}{|c|}{ Univariate analysis } & \multicolumn{2}{|c|}{ Multivariate analysis } \\
\hline & HR, (95\% Cl) & $P$-value & HR, (95\% Cl) & $P$-value \\
\hline Collaborative management & $0.30(0.07-0.84)$ & 0.018 & $0.34(0.08-0.96)$ & 0.041 \\
\hline Predicted baseline FVC <60\% & $2.32(1.27-4.18)$ & 0.007 & $2.13(1.17-3.85)$ & 0.015 \\
\hline Age $>70$ years & $0.96(0.52-1.86)$ & 0.896 & - & - \\
\hline Female sex & $1.16(0.63-2.08)$ & 0.631 & - & - \\
\hline $\mathrm{BMI}, \mathrm{kg} / \mathrm{m}^{2}$ & $1.04(0.96-1.12)$ & 0.387 & - & - \\
\hline Ex-smoker & $0.98(0.55-1.78)$ & 0.953 & - & - \\
\hline Home oxygen therapy & $1.26(0.43-2.91)$ & 0.642 & - & - \\
\hline Concomitant use of prednisolone & $1.61(0.87-2.89)$ & 0.127 & - & - \\
\hline
\end{tabular}

BMI, body mass index; FVC, forced vital capacity; HR, hazard ratio.

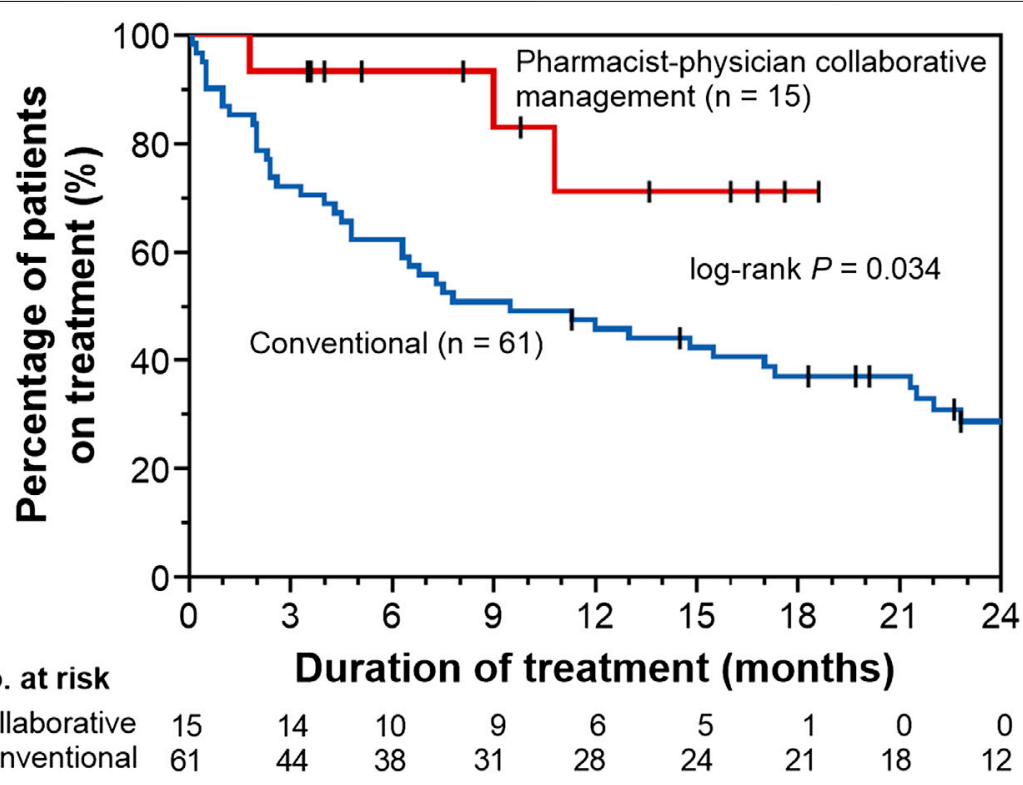

FIGURE 2 | Kaplan-Meier curves for time to drug discontinuation in the pharmacist-physician collaborative management and conventional groups. 
TABLE 3 | Patient characteristics after propensity score matching

\begin{tabular}{|c|c|c|c|}
\hline & $\begin{array}{l}\text { Collaborative } \\
\text { management } \\
(n=10)\end{array}$ & $\begin{array}{l}\text { Conventional } \\
(n=10)\end{array}$ & $P$-value \\
\hline Age, years & $76(68-79)$ & $78(71-83)$ & 0.425 \\
\hline Male, n (\%) & $7(70.0)$ & $5(50.0)$ & 0.650 \\
\hline Body weight, kg & $59.1(52.6-68.8)$ & $52.9(50.4-65.1)$ & 0.427 \\
\hline $\mathrm{BMl}, \mathrm{kg} / \mathrm{m}^{2}$ & $23.4(19.9-24.9)$ & $21.8(20.5-23.6)$ & 0.677 \\
\hline \multicolumn{4}{|l|}{ Smoker, n (\%) } \\
\hline Ex & $7(70.0)$ & $7(70.0)$ & 1.000 \\
\hline Never & $3(30.0)$ & $3(30.0)$ & \\
\hline Home oxygen therapy, n (\%) & $1(10.0)$ & $1(10.0)$ & 1.000 \\
\hline Prednisolone use, n (\%) & $5(50.0)$ & $5(50.0)$ & 0.372 \\
\hline \multicolumn{4}{|l|}{ Pulmonary function tests } \\
\hline FVC (L) & $2.4(1.6-2.8)$ & $2.2(1.8-2.4)$ & 0.520 \\
\hline FVC (\% pred) & $77.1(69.1-85.3)$ & 76.5 (53.7-88.0) & 1.000 \\
\hline DLco (\% pred) & $58.7(47.5-79.0)$ & $54.2(37.9-67.1)$ & 0.473 \\
\hline 6-min walk distance, $\mathrm{m}$ & $450(276-569)^{a}$ & $410(272-488)^{\mathrm{b}}$ & 0.773 \\
\hline
\end{tabular}

$D L C O$, diffusing capacity of carbon monoxide; FVC, forced vital capacity. Continuous data are shown as the median (interquartile range). Fisher's exact test and Wilcoxon rank sum tests were used to compare categorical and continuous data, respectively.

aMissing data from 1 patient.

${ }^{b}$ Missing data from 2 patients.

\section{DISCUSSION}

We established and evaluated an ambulatory care pharmacy practice for the pharmacist-physician collaborative management of out-patients with IPF receiving anti-fibrotic therapy. Of the 56 suggestions made by the pharmacists to the physicians, 52 (92.9\%) were accepted by the physicians. The rates of pirfenidone discontinuation owing to ADEs after 3 and 6 months of treatment tended to be lower after the initiation of the ambulatory care pharmacy practice, and the time to pirfenidone discontinuation in the collaborative management group was significantly longer than that in the conventional management group. Moreover, multivariate analysis revealed that the collaborative management approach significantly reduced the risk of pirfenidone discontinuation. This result was confirmed by a propensity score-matching analysis. To the best of our knowledge, this is the first report to demonstrate that pharmacist-physician collaborative management in an ambulatory setting successfully prevented the discontinuation of pirfenidone treatment in patients with IPF.

In real-world clinical practice, the rates of pirfenidone discontinuation owing to any reason and owing to ADEs at
12 months have been reported to be 35-75\% (Ogura et al., 2015; Bando et al., 2016; Barratt et al., 2018; Ogawa et al., 2018; Uehara et al., 2018; Sköld et al., 2019) and 15-35\% (Ogura et al., 2015; Bando et al., 2016; Galli et al., 2017; Barratt et al., 2018), respectively. In our study, the rates of pirfenidone discontinuation owing to any reason and owing to ADEs at 12 months were 51.7 vs. $33.3 \%$ and 35.0 vs. $33.3 \%$ in the conventional vs. collaborative management groups; these values were generally within the range reported previously. The multivariate analysis revealed that an $\mathrm{FVC}<60 \%$ was a risk factor for the discontinuation of pirfenidone. This result supported the findings of previous studies (Galli et al., 2017; Barratt et al., 2018; Uehara et al., 2018). On the contrary, a part of our results was not consistent with the results of previous largerscale studies. In the PASSPORT study, female sex was a significant risk factor for early treatment discontinuation due to ADEs (Cottin et al., 2018). The percentage of females was relatively higher in the conventional group than in the collaborative management group. Although we performed propensity score matching to adjust the imbalance in patient characteristics between the groups, it was difficult to completely adjust these imbalances. This was likely due to the small sample size in our study; therefore, our results should be confirmed in larger-scale studies.

The usefulness of patient-centered, multidisciplinary team care for patients with IPF has been previously assessed (Chaudhuri et al., 2014; Wuyts et al., 2014; Duck et al., 2015; Martinez and Flaherty, 2017). Duck et al. reported the effective collaboration of specialist IPF nurses with physicians and patients in the IPF Care program (Duck et al., 2015). In their review article, they described that nurses provide several supports. The IPF Care program, which is initiated when the patient is prescribed pirfenidone, involves phone calls and patienttailored information booklets. Additionally, the nurses discuss the treatment of each patient directly with the physicians. In some countries, the program also involves face-to-face visits with nurses. In our hospital, nurses have multiple important roles within the multidisciplinary IIP support team. For example, nurses advise patients and their family about their lifestyle, provide education about home oxygen therapy, and support advanced care planning processes (Rajala et al., 2016). Additionally, we have previously provided ambulatory care pharmacy practice services for patients treated with oral anticancer drugs and direct-acting antiviral agents for hepatitis C virus (Yamamoto et al., 2018). Therefore, based on our

TABLE 4 | Number of suggestions provided by the pharmacists and physicians' responses.

\section{Number of suggestions}

Required number of supportive care medicines that were previously prescribed ${ }^{\mathrm{a}}$

Additional supportive care

Others
38

Number of suggestions accepted by physicians

$35(92.1 \%)$

13

aln pharmacist-physician collaborative management, the pharmacists assessed whether the patients could appropriately take supportive care medications, such as domperidone. If the patient took the supportive medicine inadequately, the pharmacist educated the patient on the appropriate use. Then, the pharmacist suggested the number of required supportive care medicines to the physicians. 
experience, we established the ambulatory care pharmacy practice for patients receiving anti-fibrotic agents.

In our ambulatory care pharmacy practice, the pharmacists educated patients on how to take supportive care medicines upon initiation of pirfenidone treatment. At the second visit or later, the pharmacists assessed the potential ADEs and the appropriateness of the supportive care medicines; they then suggested prescriptions to the physicians. Most of the suggestions made by the pharmacists pertained to the required number of supportive care medicines that were previously prescribed (38/56 suggestions). Interestingly, although 52 of the 61 patients in the conventional care group were prescribed antiemetics, including domperidone, metoclopramide, and mosapride, the incidence of drug discontinuation owing to ADEs in these patients was not significantly decreased (Supplementary Table S1). Overall, prescribing supportive care medications alone might not be sufficient to manage ADEs, but detailed explanations are essential for each patient. Numerous studies have reported the usefulness of pharmacistinvolved team care in pharmacotherapies (Yamada and Nabeshima, 2015; Yamamoto et al., 2018; Crutzen et al., 2019; Zhang et al., 2019). Our results showed that pharmacist-physician collaborative management, in an ambulatory care setting, can also improve care for patients with IPF receiving pirfenidone.

There were some limitations to our study. First, this was a single-centered, retrospective study. Second, the number of patients in the pharmacist-physician collaborative management group was small, and the sample sizes were imbalanced between the groups. Despite performing propensity-score matching, in addition to the primary analysis, to confirm the robustness of the usefulness of our pharmacist-physician collaborative management, the sample size of the score-matched cohort was very small. Our preliminary findings should be confirmed prospectively in further studies in other institutions. Thirdly, the ideal follow-up period could not be determined from this study design. Our pharmacy practice was generally provided for all patients during the first 3 months from the start of pirfenidone treatment because the majority of dose reductions or discontinuations are observed during the early phase (Lancaster et al., 2017). However, two patients in the collaborative management group discontinued pirfenidone owing to ADEs more than 3 months after treatment initiation.

In conclusion, we established an ambulatory care pharmacy practice for the pharmacist-physician collaborative management of out-patients with IPF receiving pirfenidone treatment. The

\section{REFERENCES}

Azuma, A., Nukiwa, T., Tsuboi, E., Suga, M., Abe, S., Nakata, K., et al. (2005). Double-blind, placebo-controlled trial of pirfenidone in patients with idiopathic pulmonary fibrosis. Am. J. Respir. Crit. Care Med. 171, 1040-1047. doi:10.1164/ rccm.200404-5710C

Bando, M., Yamauchi, H., Ogura, T., Taniguchi, H., Watanabe, K., Azuma, A., et al. (2016). Clinical experience of the long-term use of pirfenidone for idiopathic pulmonary fibrosis. Intern. Med. 55 (5), 443-448. doi:10.2169/ internalmedicine. 55.5272 results of our study suggest that, compared to conventional management, collaborative management may be useful for preventing the discontinuation of pirfenidone. Our preliminary findings warrant further prospective investigations with a larger sample size.

\section{DATA AVAILABILITY STATEMENT}

The datasets generated for this study are available on request to the corresponding author.

\section{ETHICS STATEMENT}

The studies involving human participants were reviewed and approved Institutional Review Board, Kobe City Medical Center General Hospital, Kobe, Japan.

\section{AUTHOR CONTRIBUTIONS}

YS, HI, KK, RM, MK, RT, KT, and TH conceived and designed the study. YS, HI, KK, MM, RM, and MK collected the data. HI analyzed data. YS, KK, and MM verified the analyzed data. YS drafted the manuscript. HI, NM, RM, MK, RH, KN, AN, RT, KT, and $\mathrm{TH}$ revised the manuscript. KK, NM, RM, MK, RH, KN, AN, $\mathrm{RT}, \mathrm{KT}$, and $\mathrm{TH}$ interpreted data. All authors reviewed and approved the final manuscript.

\section{ACKNOWLEDGMENTS}

We thank Daichi Fujimoto (Department of Respiratory Medicine, Kobe City Medical Center General Hospital) for providing valuable advice from clinical aspect. We also thank Takeshi Morimoto (Department of Clinical Epidemiology, Hyogo College of Medicine) for providing valuable advice regarding statistical analyses.

\section{SUPPLEMENTARY MATERIAL}

The Supplementary Material for this article can be found online at: https://www.frontiersin.org/articles/10.3389/fphar.2020.529654/ full\#supplementary-material

Barratt, S. L., Mulholland, S., Al Jbour, K., Steer, H., Gutsche, M., Foley, N., et al. (2018). South-West of England's experience of the safety and tolerability pirfenidone and nintedanib for the treatment of idiopathic pulmonary fibrosis (IPF). Front. Pharmacol. 9, 1480. doi:10.3389/fphar.2018.01480

Chaudhuri, N., Duck, A., Frank, R., Holme, J., and Leonard, C. (2014). Real world experiences: pirfenidone is well tolerated in patients with idiopathic pulmonary fibrosis. Respir. Med. 108 (1), 224-226. doi:10.1016/j.rmed.2013.11.005

Costabel, U., Bendstrup, E., Cottin, V., Dewint, P., Egan, J. J. J., Ferguson, J., et al. (2014). Pirfenidone in idiopathic pulmonary fibrosis: expert panel discussion on the management of drug-related adverse events. Adv. Ther. 31 (4), 375-391. doi:10.1007/s12325-014-0112-1 
Cottin, V., Koschel, D., Günther, A., Albera, C., Azuma, A., Sköld, C. M., et al. (2018). Long-term safety of pirfenidone: results of the prospective, observational PASSPORT study. ERJ. Open. Res. 4 (4), 00084-02018. doi:10. 1183/23120541.00084-2018

Crutzen, S., Schuling, J., Hugtenburg, J. G., Verduijn, M., Teichert, M., Taxis, K., et al. (2019). Development and piloting of an algorithm to select older patients for different types of medication review. Front. Pharmacol. 10, 217. doi:10.3389/ fphar.2019.00217

Duck, A., Pigram, L., Errhalt, P., Ahmed, D., and Chaudhuri, N. (2015). IPF care: a support program for patients with idiopathic pulmonary fibrosis treated with pirfenidone in Europe. Adv. Ther. 32 (2), 87-107. doi:10.1007/s12325-0150183-7

Ferrara, G., Luppi, F., Birring, S. S., Cerri, S., Caminati, A., Sköld, M., et al. (2018). Best supportive care for idiopathic pulmonary fibrosis: current gaps and future directions. Eur. Respir. Rev. 27 (147), 170076. doi:10.1183/16000617.0076-2017

Galli, J. A., Pandya, A., Vega-Olivo, M., Dass, C., Zhao, H., and Criner, G. J. (2017). Pirfenidone and nintedanib for pulmonary fibrosis in clinical practice: tolerability and adverse drug reactions. Respirology 22 (6), 1171-1178. doi:10.1111/resp.13024

Homma, S., Bando, M., Azuma, A., Sakamoto, S., Sugino, K., Ishii, Y., et al. (2018). Japanese guideline for the treatment of idiopathic pulmonary fibrosis. Respir. Investig. 56, 268-291. doi:10.1016/j.resinv.2018.03.003

Hughes, G., Toellner, H., Morris, H., Leonard, C., and Chaudhuri, N. (2016). Real world experiences: pirfenidone and nintedanib are effective and well tolerated treatments for idiopathic pulmonary fibrosis. J. Clin. Med. 5 (9), 78. doi:10. 3390/jcm5090078

Iwata, T., Yoshino, I., Yoshida, S., Ikeda, N., Tsuboi, M., Asato, Y., et al. (2016). A phase II trial evaluating the efficacy and safety of perioperative pirfenidone for prevention of acute exacerbation of idiopathic pulmonary fibrosis in lung cancer patients undergoing pulmonary resection: west Japan Oncology Group 6711 L (PEOPLE Study). Respir. Res. 17 (1), 90. doi:10.1186/s12931-016-0398-4

King, T. E., Jr., Bradford, W. Z., Castro-Bernardini, S., Fagan, E. A., Glaspole, I., Glassberg, M. K., et al. (2014). A phase 3 trial of pirfenidone in patients with idiopathic pulmonary fibrosis. N. Engl. J. Med. 370, 2083-2092. doi:10.1056/ NEJMoa1402582

Lancaster, L., Albera, C., Bradford, W. Z., Costabel, U., du Bois, R. M., Fagan, E. A., et al. (2016). Safety of pirfenidone in patients with idiopathic pulmonary fibrosis: integrated analysis of cumulative data from 5 clinical trials. BMJ. Open. Respir. Res. 3 (1), e000105. doi:10.1136/bmjresp-2015-000105

Lancaster, L. H., de Andrade, J. A., Zibrak, J. D., Padilla, M. L., Albera, C., Nathan, S. D., et al. (2017). Pirfenidone safety and adverse event management in idiopathic pulmonary fibrosis. Eur. Respir. Rev. 26 (146), 170057. doi:10. 1183/16000617.0057-2017

Lederer, D. J., and Martinez, F. J. (2018). Idiopathic pulmonary fibrosis. N. Engl. J. Med. 378, 1811-1823. doi:10.1056/NEJMra1705751

Ley, B., Collard, H. R., and King, T. E., Jr. (2011). Clinical course and prediction of survival in idiopathic pulmonary fibrosis. Am. J. Respir. Crit. Care Med. 183, 431-440. doi:10.1164/rccm.201006-0894CI

Martinez, F. J., and Flaherty, K. R. (2017). Comprehensive and individualized patient care in idiopathic pulmonary fibrosis: refining approaches to diagnosis, prognosis, and treatment. Chest 151 (5), 1173-1174. doi:10.1016/j.chest.2017. 03.017

Noble, P. W., Albera, C., Bradford, W. Z., Costabel, U., du Bois, R. M., Fagan, E. A., et al. (2016). Pirfenidone for idiopathic pulmonary fibrosis: analysis of pooled data from three multinational phase 3 trials. Eur. Respir. J. 47 (1), 243-253. doi:10.1183/13993003.00026-2015

Noble, P. W., Albera, C., Bradford, W. Z., Costabel, U., Glassberg, M. K., Kardatzke, D., et al. (2011). Pirfenidone in patients with idiopathic pulmonary fibrosis
(CAPACITY): two randomised trials. Lancet. 377 (9779), 1760-1769. doi:10. 1016/S0140-6736(11)60405-4

Ogawa, K., Miyamoto, A., Hanada, S., Takahashi, Y., Murase, K., Mochizuki, S., et al. (2018). The efficacy and safety of long-term pirfenidone therapy in patients with idiopathic pulmonary fibrosis. Intern. Med. 57 (19), 2813-2818. doi:10.2169/internalmedicine.0559-17

Ogura, T., Azuma, A., Inoue, Y., Taniguchi, H., Chida, K., Bando, M., et al. (2015). All-case post-marketing surveillance of 1371 patients treated with pirfenidone for idiopathic pulmonary fibrosis. Respir. Investig. 53 (5), 232-241. doi:10.1016/ j.resinv.2015.06.001

Raghu, G., Rochwerg, B., Zhang, Y., Garcia, C. A. C., Azuma, A., Behr, J., et al. (2015). An official ATS/ERS/JRS/ALAT clinical practice guideline: treatment of idiopathic pulmonary fibrosis. An update of the 2011 clinical practice guideline. Am. J. Respir. Crit. Care Med. 192 (2), e3-e19. doi:10.1164/rccm.201506-1063ST

Rajala, K., Lehto, J. T., Saarinen, M., Sutinen, E., Saarto, T., and Myllärniemi, M. (2016). End-of-life care of patients with idiopathic pulmonary fibrosis. BMC Palliat. Care 15, 85. doi:10.1186/s12904-016-0158-8

Sköld, C. M., Arnheim-Dahlström, L., Bartley, K., Janson, C., Kirchgaessler, K.-U., Levine, A., et al. (2019). Patient journey and treatment patterns in adults with IPF based on health care data in Sweden from 2001 to 2015. Respir. Med. 155, 72-78. doi:10.1016/j.rmed.2019.06.001

Taniguchi, H., Ebina, M., Kondoh, Y., Ogura, T., Azuma, A., Suga, M., et al. (2010). Pirfenidone in patients with idiopathic pulmonary fibrosis. Eur. Respir. J. 35 (4), 821-829. doi:10.1183/09031936.00005209

Uehara, M., Enomoto, N., Oyama, Y., Suzuki, Y., Kono, M., Furuhashi, K., et al. (2018). Body size-adjusted dose analysis of pirfenidone in patients with interstitial pneumonia. Respirology 23 (3), 318-324. doi:10.1111/resp.13145

Wuyts, W. A., Peccatori, F. A., and Russell, A.-M. (2014). Patient-centred management in idiopathic pulmonary fibrosis: similar themes in three communication models. Eur. Respir. Rev. 23 (132), 231-238. doi:10.1183/ 09059180.00001614

Yamada, K., and Nabeshima, T. (2015). Pharmacist-managed clinics for patient education and counseling in Japan: current status and future perspectives. J. Pharm. Health. Care. Sci. 1 (1), 2. doi:10.1186/s40780-014-0001-4

Yamamoto, H., Ikesue, H., Ikemura, M., Miura, R., Fujita, K., Chung, H., et al. (2018). Evaluation of pharmaceutical intervention in direct-acting antiviral agents for hepatitis $\mathrm{C}$ virus infected patients in an ambulatory setting: a retrospective analysis. J. Pharm. Health. Care. Sci. 4 (1), 17. doi:10.1186/ s40780-018-0113-3

Zhang, J., Qian, X., Zhang, L., Hu, L., Fan, L., Wang, Q., et al. (2019). Evaluation of the effectiveness of clinical pharmacists' consultation in the treatment of infectious diseases: a single-arm, prospective cohort study. Front. Pharmacol. 10, 187. doi:10.3389/fphar.2019.00187

Conflict of Interest: KT received lecture fee from Shionogi and Co., Ltd.

The remaining authors declare that the research was conducted in the absence of any commercial or financial relationships that could be construed as a potential conflict of interest.

Copyright (c) 2020 Satsuma, Ikesue, Kusuda, Maeda, Muroi, Mori, Kogo, Hirabayashi, Nagata, Nakagawa, Tachikawa, Tomii and Hashida. This is an open-access article distributed under the terms of the Creative Commons Attribution License (CC BY). The use, distribution or reproduction in other forums is permitted, provided the original author(s) and the copyright owner(s) are credited and that the original publication in this journal is cited, in accordance with accepted academic practice. No use, distribution or reproduction is permitted which does not comply with these terms. 